\title{
Stenotrophomonas maltophilia Meningitis - A Case Series and Review of the Literature
}

\author{
Iffat Khanum $^{1}$, Aisha Ilyas ${ }^{1}$, Farheen Ali ${ }^{2}$ \\ PAK \\ Corresponding author: Iffat Khanum, iffat.khanum.ik@gmail.com
}

1. Infectious Diseases, Aga Khan University Hospital, Karachi, PAK 2. Medicine, Aga Khan University Hospital, Karachi,

\begin{abstract}
Stenotrophomonas maltophilia is an infrequent cause of acute bacterial meningitis and only a few cases have been reported in the literature. Infection is associated with morbidity and mortality, and its optimal management remains ill-defined. The aim of the current study is to review the management of S. maltophilia meningitis.
\end{abstract}

We described two cases of $S$. maltophilia meningitis following neurosurgical procedures. The first patient was a 60-year-old female. She was admitted to the hospital with a left basal ganglia bleed and underwent placement of an external ventricular drain for the treatment of hydrocephalus. She developed S. maltophilia meningitis 20 days after surgery. She was successfully treated with a combination of trimethoprimsulfamethoxazole and intravenous colistin and the removal of the drain. She successfully underwent a ventriculoperitoneal (VP) shunt placement at the therapeutic midway point. The second patient was a 35year-old male with a history of intracranial aneurysm bleeding. He had undergone a craniotomy and placement of a ventriculoperitoneal shunt two years previously. His shunt was replaced twice due to blockage. The last replacement had occurred 15 days prior to the development of meningitis. He was treated with a combination of trimethoprim-sulfamethoxazole and ceftazidime (as well as undergoing another shunt replacement) and experienced an excellent recovery.

S. maltophilia is a rare but important cause of nosocomial meningitis. It is strongly associated with prior hospitalization and neurosurgical intervention, which is also found in our case series. The management of S. maltophilia meningitis is a therapeutic challenge due to its high resistance to multiple antibiotics. Optimal therapy is based on antimicrobial sensitivity, and the trimethoprim-sulfamethoxazole-based combination has been shown to be successful. The duration of therapy is debatable, but like most gram-negative meningitis infections, therapy lasting up to three weeks appears to be adequate.

Review began 09/21/2020 Review ended 10/28/2020 Published 10/28/2020

\section{(c) Copyright 2020}

Khanum et al. This is an open access article distributed under the terms of the Creative Commons Attribution License CC-BY 4.0., which permits unrestricted use, distribution, and reproduction in any medium, provided the original author and source are credited.
Categories: Neurology, Infectious Disease, Neurosurgery

Keywords: nosocomial meningitis, stenotrophomonas meningitis, gram-negative meningitis

\section{Introduction}

Stenotrophomonas maltophilia, initially known as Bacterium bookeri, was first isolated in 1943. It was periodically classified as a member of the genus Pseudomonas, then Xanthomonas, and finally Stenotrophomonas in 1993. The genus Stenotrophomonas currently consists of four species, only one of which is known to cause infection in humans. S. maltophilia is an aerobic, non-fermentative, gram-negative bacillus that can occur in almost any aquatic or humid environment, including drinking water supplies [1]. S.maltophilia, through its ability to form a biofilm, can easily adhere to the devices and surfaces in a hospital environment which not only helps in its transmission but also increases its resistance to many antibiotics.

Historically considered as an opportunistic pathogen, S. maltophilia is now recognized as an important nosocomial pathogen with a high mortality rate. Infections due to S. maltophilia are particularly common with immunosuppression, extended use of antibiotics, intensive care unit admission, prolonged hospital stay, and presence of indwelling devices. It is responsible for a range of infections like pneumonia, bacteremia, biliary sepsis, urinary tract infection, endocarditis, meningitis, skin and soft tissue infection, endophthalmitis, septicemia, catheter-related bloodstream infection, osteomyelitis, and joint infections [2].

Despite the fact that $S$. maltophilia can cause a variety of infections, it still remains an uncommon cause of meningitis [3]. Its management is challenging due to the resistance of $S$. maltophilia to multiple antimicrobial agents and different antimicrobial susceptibility among different strains. It is only reported in the form of brief case series or case reports in the literature [4].

We report two cases of $S$. maltophilia meningitis following neurosurgical interventions and discuss therapeutic advances based on a brief review of the literature. 


\section{Cureus}

\section{Case Presentation}

\section{Case one}

A 60-year-old female with uncontrolled hypertension, presented with basal ganglia bleed and obstructive hydrocephalus. She underwent emergency placement of an external ventricular drain (EVD). Twenty days following the procedure, she developed a fever with no overt systemic signs of infection. As part of the fever evaluation, a cerebrospinal fluid (CSF) sample was sent for analysis and culture. Her initial CSF analysis showed very low glucose levels and polymorphic pleocytosis with gram-negative bacilli seen on the CSF gram stain, thus confirming meningitis (Table 1). The gram-negative bacilli were later identified as $S$. maltophilia using the 5 analytical profile index (API) 20NE test. API 20NE is a biochemical panel for identification and differentiation of non-fastidious Gram-negative rods not belonging to the family Enterobacteriaceae. It is a well-established method for manual microorganism identification to the species level. Considering the high prevalence of multidrug-resistant gram-negative organisms like MDR Acinetobacter and Klebsiella spp. in our hospital, the patient was empirically started on intravenous meropenem (2 gm every eight hours) and polymyxin E (3 million units every eight hours) along with intrathecal polymyxin E (300,000 IU/day). Drug susceptibility on disc diffusion showed that the organism was only sensitive to trimethoprim-sulfamethoxazole (TMP-SXM) and colistin. Therefore, her treatment was modified on the third day of illness. Intravenous meropenem was stopped, colistin (both intravenous and intrathecal) was continued and TMP-SMX $(15 \mathrm{~m} / \mathrm{kg} / \mathrm{d}$ in three divided doses) was added. The patient underwent replacement of the EVD after four days of the modified therapy. Three days following the removal of the EVD and seven days after the start of the TMP-SMX-based combination regimen, her repeat CSF cultures were negative, with significant improvement in all the CSF indices (Table 1).

\begin{tabular}{|c|c|c|c|}
\hline CSF Analysis & DAY 1 & DAY 4 & DAY 7 \\
\hline Glucose (mg/dl) & 12 & 84 & 76 \\
\hline Protein (mg/dl) & 88 & 78 & 47 \\
\hline WBC ( $0-5)$ & 80 & 26 & 12 \\
\hline DLC \% & $90 \%$ neutrophils & $10 \%$ neutrophils & $5 \%$ neutrophils \\
\hline Culture & S. maltophilia & No growth & No growth \\
\hline
\end{tabular}

\section{TABLE 1: Cerebrospinal fluid analysis Case 1}

Normal values in cerebrospinal fluid (CSF); Glucose 40-70 mg/dl, Protein 15-40 mg/dl, WBC: white blood cell 0-5 ,

DLC Differential leucocytes count

The intrathecal antibiotics were stopped once the CSF culture (day seven) was negative, but systemic colistin was continued for two weeks and TMP-SMX for 21 days. Her initial blood cultures were reported as negative and all 11 of her subsequent CSF cultures remained negative. There was no neurological sequelae posttreatment and she successfully underwent a ventriculoperitoneal (VP) shunt placement at the therapeutic midway point.

\section{Case two}

A 35-year-old male underwent a craniotomy and VP shunt placement following an intracranial aneurysm bleed that had occurred two years previously. He required replacement of the VP shunt twice due to shunt blockage. The first replacement had been performed one year before and the second was performed two weeks prior to the onset of fever. He developed a fever with altered mental status i.e. drowsiness two weeks after the replacement of the last VP shunt. As part of the fever workup, his blood, urine, and CSF samples were sent for culture and analysis. His CSF gram stain showed gram-negative bacilli with very low CSF glucose levels, high protein levels and white cells, thus confirming a meningitis diagnosis (Table 2). 


\section{Cureus}

\begin{tabular}{|c|c|c|c|}
\hline CSF Analysis & DAY 1 & DAY 4 & DAY 15 \\
\hline Glucose (mg/dl) & 16 & 23 & 78 \\
\hline Protein( mg/dl) & 257 & 102 & 42 \\
\hline WBC $(0-5)$ & 131 & 75 & 10 \\
\hline DLC \% & $80 \%$ neutrophils & $70 \%$ neutrophils & $5 \%$ neutrophils \\
\hline Culture & S. maltophilia & No growth & No growth \\
\hline
\end{tabular}

\section{TABLE 2: Cerebrospinal fluid analysis Case 2}

Normal values in cerebrospinal fluid (CSF); Glucose $40-70 \mathrm{mg} / \mathrm{dl}$, Protein 15-40 mg/dl, WBC: white blood cell 0-5

DLC Differential leucocytes count

The bacilli were identified as S. maltophilia using the API 20NE test. The drug susceptibility on disc diffusion showed sensitivity to TMP-SMX and ceftazidime. He was initially started on intravenous meropenem (2 gm every eight hours) along with intravenous colistin, which was later modified to intravenous TMP-SMX $15 \mathrm{mg} / \mathrm{kg} / \mathrm{d}$ and ceftazidime (2 gm every eight hours) as per antibiotics sensitivities of $S$. maltophilia reported in CSF culture. Additional therapeutic measures included shunt removal. His initial blood cultures were reported negative after seven days. Clinical improvement was noted after 72 hours of treatment. Repeated CSF analyses showed gradual improvement, and all subsequent CSF cultures were negative (Table 2). VP shunt was replaced after two weeks of appropriate antibiotic therapy. He received therapy for 21 days with marked clinical recovery.

\section{Discussion}

Based on different inclusion studies, the incidence of post neurosurgical meningitis has been reported to be between $0.3 \%-8.9 \%$. Risk factors include neurosurgical procedures such as craniotomies, especially in the presence of EVD devices, prolonged hospital stays, prior antibiotic exposure, and the existence of chronic illnesses such as diabetes and malignancies. Among other gram-negative pathogens such as Escherichia coli, Acinetobacter spp., Pseudomonas aeruginosa, and Klebsiella pneumoniae, S. maltophilia remains a rare cause of healthcare-associated meningitis and only a few cases have been reported to date [4]. We performed a comprehensive literature review to identify cases of S. maltophilia meningitis (Table 3).

\begin{tabular}{|c|c|c|c|c|c|}
\hline Year of publication & Age/gender & $\begin{array}{l}\text { Prior Neurosurgical } \\
\text { interventions }\end{array}$ & Comorbid illnesses & Therapy (Duration ) & Outcome \\
\hline Patrick et al 1975 [5] & 70 years $/ \mathrm{M}$ & None & Emphysema & Sulphamidine and chloramphenicol (12 days) & Cured \\
\hline Denis et al, 1977 [6] & $\begin{array}{l}13 \\
\text { months/F }\end{array}$ & None & None & Chloramphenicol and sulfadoxime/duration ${ }^{\star}$ & Cured \\
\hline Denis et al, 1977 [6] & 8 months/M & None & None & Ampicillin and colistin ${ }^{\star}$ & Died \\
\hline Trump et al, 1982 [7] & 52 years/F & Ommaya reservoir & $\begin{array}{l}\text { Metastatic } \\
\text { malignancy }\end{array}$ & Chloramphenicol and gentamicin ${ }^{\star}$ & Cured \\
\hline Dewi et al, 1984 [8] & $\begin{array}{l}\text { Preterm } \\
\text { infant/M }\end{array}$ & Yes & IVH & none & Died \\
\hline Muder et al, 1987 [9] & 65 years/M & EVD & IVH & TMP-SXM ${ }^{*}$ & Cured \\
\hline $\begin{array}{l}\text { Girijaratnakum-ari et al, } \\
1993[10]\end{array}$ & 28 years/F & craniotomy & $\mathrm{CP}$ angle tumor & I/V ciprofloxacin (3 weeks) & Cured \\
\hline Nguyen et al,1994 [11] & 64 years/M & VPS & Meningioma & I/V TMP-SX with intrathecal gentamicin & Cured \\
\hline $\begin{array}{l}\text { Papadakis et al, } 1997 \\
\text { [12] }\end{array}$ & 36 years/F & Ommaya reservoir & Melanoma & TMP-SXM* ${ }^{*}$ & Cured \\
\hline $\begin{array}{l}\text { Papadakis et al, } 1997 \\
\text { [12] }\end{array}$ & 41 years/M & Ommaya reservoir & Autologous SCT & TMP-SXM $^{*}$ & Died \\
\hline
\end{tabular}




\section{Cureus}

\begin{tabular}{|c|c|c|c|c|c|}
\hline Spencer et al, 2001 [13] & 31 years/F & No & None & TMP-SXM (14 days) & Cured \\
\hline Caylan et al,2002 [14] & 52 years $/ \mathrm{M}$ & VPS & SAH & TMP-SXM DS (3 weeks) & Cured \\
\hline $\begin{array}{l}\text { Platsouka et al, } 2002 \\
\text { [15] }\end{array}$ & 42 years/M & Yes & Malignancy & TMP-SXM with ceftazidime * & Cured \\
\hline Lo et al, 2002 [16] & $\begin{array}{l}\text { Preterm } \\
\text { infant } / F\end{array}$ & No & None & Ciprofloxacin (4 weeks) & Cured \\
\hline $\begin{array}{l}\text { Libanore et al, } 2004 \\
\text { [17] }\end{array}$ & 49 years/M & No & None & Amikacin with ceftazidime (14 days ) & Cured \\
\hline Reddy et al , 2006 [18] & 48 years $/ \mathrm{M}$ & craniotomy & Pituitary tumor & $\begin{array}{l}\text { TMP-SXM ( } 12 \text { days )followed by } \\
\text { moxifloxacin ( } 21 \text { days) }\end{array}$ & Cured \\
\hline $\begin{array}{l}\text { Yemisen et al , } 2008 \\
\text { [2] }\end{array}$ & 30 years $/ \mathrm{M}$ & Craniotomy & $\begin{array}{l}\text { Subdural } \\
\text { hematoma }\end{array}$ & Ciprofloxacin and TMP-SXM (14 days) & Cured \\
\hline Rojas et al , 2009 [19] & Preterm/M & Yes & Nil & Ciprofloxacin and TMP-SXM (21 days) & Cured \\
\hline Huang et al ,2013 [4] & 48 years $/ \mathrm{M}$ & Craniotomy and EVD & Nil & IV TMP-SXM + IV ciprofloxacin (23 days) & Cured \\
\hline Huang et al ,2013 [4]. & 61 years/M & craniotomy and EVD & Nil & $\begin{array}{l}\text { IV ceftazidime (63 days }) \rightarrow \text { IV ciprofloxacin } \\
(35 \text { days ) }\end{array}$ & Cured \\
\hline Huang et al , 2013 [4]. & 43 years/F & craniotomy and EVD & $\begin{array}{l}\text { Intracranial artery } \\
\text { aneurysm }\end{array}$ & $\begin{array}{l}\text { Oral TMP-SXM (33) + IV levofloxacin (11) } \rightarrow \text { IV } \\
\text { ciprofloxacin (22) }\end{array}$ & Cured \\
\hline Huang et al,2013 [4] & 48 years $/ F$ & craniotomy and EVD & $\begin{array}{l}\text { intracranial artery } \\
\text { aneurysm }\end{array}$ & IV TMP - SXM + IV moxifloxacin (42 days) & Cured \\
\hline Huang et al, 2013 [4] & 62 years/F & craniotomy and EVD & DM & IV SMZ-TMP (61)+ IV moxifloxacin (42) (28) & Died \\
\hline Huang et al,2013 [4] & 45 years $/ \mathrm{M}$ & $\begin{array}{l}\text { craniotomy and VP } \\
\text { shunt }\end{array}$ & Pituitary tumor & $\begin{array}{l}\text { IV moxifloxacin } \rightarrow \text { IV moxifloxacin+ oral } \\
\text { TMP-SXM (14) }\end{array}$ & Died \\
\hline Wang et al, $2013[3]$ & 73 years $/ \mathrm{M}$ & Yes, EVD & Nil & Ceftazidime (21 days) & Cured \\
\hline Wang et al, 2013 [3] & 61 years $/ \mathrm{M}$ & Neuro endoscopy & Nil & Ceftazidime (21 days) & Cured \\
\hline Correia et al, 2014 [20] & 4 years/M & EVD & Nil & $\begin{array}{l}\text { TMP-SMX + ceftazidime + levofloxacin } 21 \\
\text { days }\end{array}$ & Cured \\
\hline Ibrahim et al,2018 [1] & $\begin{array}{l}13 \text { days } \\
\text { old/M }\end{array}$ & No & Nil & TMP-SMX and Ciprofloxacin (21 days) & Cured \\
\hline Present case & 35 years $/ \mathrm{M}$ & Yes $/$ P shunt & Nil & TMP-SMX and ceftazidime (21 days) & Cured \\
\hline Present case & 60 years/F & Yes/EVD & HTN & TMP-SMX and colistin - (21 days) & Cured \\
\hline
\end{tabular}

\section{TABLE 3: . Cases with S. maltophilia meningitis}

F: female, M: male, TMP-SXM: Trimethoprim- sulfamethoxazole, IVH: intraventricular haemorrhage, SCT: stem cell transplant, EVD: external ventricular drain, VPS: ventriculo peritoneal shunt, HTN: hypertension, SAH: subarachnoid haemorrhage *duration not known

Among the 30 reported cases of $S$. maltophilia meningitis, there was a male predominance (67.7\%) and only seven cases (22.5\%) belonged to pediatric age groups. In the current review, $87 \%$ of $S$. maltophilia meningitis cases were hospital-acquired and a majority of patients had undergone neurosurgical intervention prior to the onset of meningitis. Prolonged antibiotic therapy (e.g. carbapenem, antipseudomonal cephalosporin, and aminoglycosides ), healthcare exposure, and prior neurosurgical procedures were the major risk factors (in more than $50 \%$ of the cases) associated with S. maltophilia infection in healthcare settings [4]. Clinicians should suspect $S$. maltophilia meningitis in patients with a previous history of hospitalization, presence of devices like VP shunt, neurosurgical procedure, and prior prolonged antibiotic exposure.

The management of infections caused by S. maltophilia is challenging due to its intrinsic resistance to multiple antibiotics, the difficulty of in vitro testing antimicrobial susceptibility, and the development of resistance during treatment. S. maltophilia has multiple mechanisms of resistance including the production of beta-lactamase, decreased membrane permeability to antibiotics, the presence of efflux pumps, and antibiotic modifying enzymes [11]. The optimal antibiotics to treat S. maltophilia infections are based on in vitro studies, anecdotal evidence, and only a few clinical trials. 
The antibiotic of choice for $S$. maltophilia is TMP-SMX, which is based on good clinical outcomes and susceptibility profiles reported from previous studies $[2,4]$. Furthermore, TMP-SMX is the most frequently used antibiotic, both alone or in combination, in previously reported cases of $S$. maltophilia meningitis $[1,2,4,9,11-15,18-20]$. Both of our patients were treated with TMP-SMX (in combination with ceftazidime in one patient, and colistin in the other) based on susceptibility data.

Other antibiotics reportedly used in combination with TMP-SMX for S. maltophilia meningitis are quinolones (ciprofloxacin, levofloxacin, and moxifloxacin), ceftazidime, and polymyxin. Among the 19 cases from the current review that were treated with TMP-SMX, the majority received combination therapy.

There is no guideline available for the management of severe infection caused by S. maltophilia. Due to its resistance to multiple antibiotics, TMP-SMX is used in combination with other antimicrobial agents for the treatment of severe infection; although, there is lack of clinical data regarding the efficacy of combination therapy [2]. There was no difference found in the clinical outcomes of patients with S. maltophilia meningitis, either when treated with TMP-SMX alone or in combination therapy. The duration of therapy was between 14 and 21 days. Preferably, devices like EVD, VP shunt should be removed to achieve microbiological clearance.

The overall mortality rate of patients with $S$. maltophilia meningitis was $16 \%$ in the present review but high mortality was observed in pediatric patients in comparison to adults ( $28.5 \%$ vs $12.5 \%)$. S. maltophilia meningitis poses a treatment challenge due to the limited number of effective antibiotics available.

To the best of our knowledge, this is the first case series of $S$. maltophilia meningitis from our country. Both cases were managed successfully with antibiotics along with replacement of intraventricular devices and had good clinical outcomes.

\section{Conclusions}

S. maltophilia is an extremely rare but emerging cause of nosocomial meningitis and is strongly associated with prior hospitalization, prolonged use of antibiotics, presence of devices, and neurosurgical intervention. The optimal therapy for $S$. maltophilia meningitis is not well defined and remains a therapeutic challenge. Trimethoprim-sulfamethoxazole is the drug of choice and duration of therapy is 14 to 21 days. TMP-SMXbased combination therapy depending upon antimicrobial susceptibility data is recommended for $S$. maltophilia meningitis with good clinical outcome. The overall mortality rate of patients with S. maltophilia meningitis was low in this review, with pediatric patients having high mortality in comparison to adults.

S. maltophilia must be considered as a potential pathogen in cases of nosocomial meningitis developed after neurosurgical intervention, prolonged hospitalization, antibiotic use, and presence of indwelling devices.

\section{Additional Information \\ Disclosures}

Human subjects: Consent was obtained by all participants in this study. Conflicts of interest: In compliance with the ICMJE uniform disclosure form, all authors declare the following: Payment/services info: All authors have declared that no financial support was received from any organization for the submitted work. Financial relationships: All authors have declared that they have no financial relationships at present or within the previous three years with any organizations that might have an interest in the submitted work. Other relationships: All authors have declared that there are no other relationships or activities that could appear to have influenced the submitted work.

\section{References}

1. Ibrahim J, Hamwi N, Rabei H, Abdelghafar M, Al-Dulaimi Z, Al Tatari H: Stenotrophomonas maltophilia meningitis in a term healthy neonate: a case report and literature review. Case Rep Ped. 2018, 2018:1-3. $10.1155 / 2018 / 1543934$

2. Yemisen M, Mete B, Tunali Y, Yentur E, Ozturk R: A meningitis case due to Stenotrophomonas maltophilia and review of the literature. Int J Infect Dis. 2008, 12:125. 10.1016/j.ijid.2008.03.028

3. Wang C-H, Hsu S-W, Tsai T-H, Wang N-C: An outbreak of trimethoprim/sulfamethoxazole-resistant Stenotrophomonas maltophilia meningitis associated with neuroendoscopy. J Med Sci. 2014, 34:235 - 237. 10.4103/1011-4564.143653

4. Huang C-R, Chen S-F, Tsai N-W, et al.: Clinical characteristics of Stenotrophomonas maltophilia meningitis in adults: a high incidence in patients with a postneurosurgical state, long hospital staying and antibiotic use. Clin Neurol Neurosurg. 2013, 115:1709 - 1715. 10.1016/j.clineuro.2013.03.006

5. Patrick S, Hindmarch J, Hague R, Harris D: Meningitis caused by Pseudomonas maltophilia . J Clin Pathol. 1975, 28:741-743.

6. Denis F, Sow A, David M, Chiron J, Samb A, Diop Mar I: Study of 2 cases of Pseudomonas maltophilia meningitis observed in Senegal [Article in French]. Bull Soc Med Afr Noire Lang Fr. 1977, 22:135-139.

7. Trump DL, Grossman SA, Thompson G, Murray K: CSF infections complicating the management of 
neoplastic meningitis: clinical features and results of therapy. Arch Intern Med. 1982, 142:583-586. 10.1001/archinte.1982.00340160163029

8. Sarvamangala Dewi J, Venkatesh A, Shivananda P: Neonatal infections due to Pseudomonas maltophilia . Indian Pediatr. 1984, 21:72-74.

9. Muder RR, Victor LY, Dummer JS, Vinson C, Lumish RM: Infections caused by Pseudomonas maltophilia: expanding clinical spectrum. Arch Intern Med. 1987, 147:1672-1674.

10.1001/archinte.1987.00370090148025

10. Girijaratnakumari T, Raja A, Ramani R, Antony B, Shivananda P: Meningitis due to Xanthomonas maltophilia. J Postgrad Med. 1993, 39:153-155.

11. Nguyen MH, Muder RR: Meningitis due to Xanthomonas maltophilia: case report and review. Clin Infect Dis. 1994, 19:325-326. 10.1093/clinids/19.2.325

12. Papadakis KA, Vartivarian SE, Vassilaki ME, Anaissie EJ: Stenotrophomonas maltophilia meningitis: report of two cases and review of the literature. J Neurosurg. 1997, 87:106-108. 10.3171/jns.1997.87.1.0106

13. Spencer MT, Baron BJ: Stenotrophomonas maltophilia meningitis: a rare cause of headache in the ED . Am J Emerg Med. 2001, 19:532-533. 10.1053/ajem.2001.27149

14. Caylan R, Aydin K, Koksal I: Meningitis caused by Stenotrophomonas maltophilia: case report and review of the literature. Ann Saudi Med. 2002, 22:216-218. 10.5144/0256-4947.2002.216

15. Platsouka E, Routsi C, Paniara O, Roussos C, Dimitriadou E, Chalkis A: Stenotrophomonas maltophilia meningitis, bacteremia and respiratory infection. Scand J Infect Dis. 2002, 34:391-392. 10.1080/00365540110080520

16. Lo W-T, Wang C-C, Lee C-M, Chu M-L: Successful treatment of multi-resistant Stenotrophomonasmaltophilia meningitis with ciprofloxacin in a pre-term infant. Eur J Pediatr. 2002, 161:680-682. 10.1007/s00431-002-1095-5

17. Libanore M, Bicocchi R, Pantaleoni M, Ghinelli F: Community-acquired infection due to Stenotrophomonas maltophilia: a rare cause of meningitis. Int J Infect Dis. 2004, 8:317-319. 10.1016/j.ijid.2004.05.002

18. Reddy P, Das S, Chandler JP, Noskin GA: Stenotrophomonas maltophilia meningitis treated with moxifloxacin: a case report and review of the literature. Infect Dis Clin Prac. 2006, 14:173-176. 10.1097/01.idc.0000194059.14537.e5

19. Rojas P, Garcia E, Calderón GM, Ferreira F, Rosso M: Successful treatment of Stenotrophomonas maltophilia meningitis in a preterm baby boy: a case report. J Med Case Rep. 2009, 3:1 - 3. 10.4076/1752-1947-3-7389

20. Correia CR, Ferreira ST, Nunes P: Stenotrophomonas maltophilia: rare cause of meningitis . Ped Int. 2014, 56:21-22. 10.1111/ped.12352 